\title{
Validation of the FIB4 index in a Japanese nonalcoholic fatty liver disease population
}

\author{
Yoshio Sumida', Masato Yoneda ${ }^{2}$, Hideyuki Hyogo ${ }^{3}$, Yoshito Itoh ${ }^{4}$, Masafumi Ono ${ }^{5 *}$, Hideki Fujii ${ }^{6}$, Yuichiro Eguchi', \\ Yasuaki Suzuki ${ }^{8}$, Noriaki Aoki ${ }^{9}$, Kazuyuki Kanemasa', Koji Fujita ${ }^{2}$, Kazuaki Chayama ${ }^{3}$, Toshiji Saibara ${ }^{5}$, \\ Norifumi Kawada ${ }^{6}$, Kazuma Fujimoto ${ }^{7}, Y_{\text {Y }}$ utaka Kohgo ${ }^{8}$, Toshikazu Yoshikawa ${ }^{4}$ and Takeshi Okanoue ${ }^{10}$, for \\ Japan Study Group of Nonalcoholic Fatty Liver Disease (JSG-NAFLD)
}

\begin{abstract}
Background: A reliable and inexpensive noninvasive marker of hepatic fibrosis is required in patients with nonalcoholic fatty liver disease (NAFLD). FIB4 index (based on age, aspartate aminotransferase [AST] and alanine aminotransferase [ALT] levels, and platelet counts) is expected to be useful for evaluating hepatic fibrosis. We validated the performance of FIB4 index in a Japanese cohort with NAFLD.

Methods: The areas under the receiver operating characteristic curves (AUROC) for FIB4 and six other markers were compared, based on data from 576 biopsy-proven NAFLD patients. Advanced fibrosis was defined as stage 34 fibrosis. FIB4 index was assessed as: age $(\mathrm{yr}) \times \mathrm{AST}(\mathrm{IU} / \mathrm{L}) /\left(\right.$ platelet count $\left.\left(10^{9} / \mathrm{L}\right) \times \sqrt{ } \mathrm{ALT}(\mathrm{IU} / \mathrm{L})\right)$

Results: Advanced fibrosis was found in 64 (11\%) patients. The AUROC for FIB4 index was superior to those for the other scoring systems for differentiating between advanced and mild fibrosis. Only 6 of 308 patients with a FIB4 index below the proposed low cut-off point $(<1.45)$ were under-staged, giving a high negative predictive value of $98 \%$. Twenty-eight of 59 patients with a FIB4 index above the high cut-off point (>3.25) were over-staged, giving a low positive predictive value of 53\%. Using these cutoffs, $91 \%$ of the 395 patients with FIB-4 values outside 1.453.25 would be correctly classified. Implementation of the FIB4 index in the Japanese population would avoid 58\% of liver biopsies.
\end{abstract}

Conclusion: The FIB4 index was superior to other tested noninvasive markers of fibrosis in Japanese patients with NAFLD, with a high negative predictive value for excluding advanced fibrosis. The small number of cases of advanced fibrosis in this cohort meant that this study had limited power for validating the high cut-off point.

\section{Background}

Type 2 diabetes mellitus is associated with nonalcoholic fatty liver disease (NAFLD) in clinical practice. NAFLD includes a wide spectrum of liver diseases ranging from simple steatosis, which is usually a benign and non-progressive condition, to nonalcoholic steatohepatitis (NASH), which can progress to liver cirrhosis (LC) and hepatocellular carcinoma (HCC) in the absence of significant alcohol consumption [1-4]. Liver biopsy remains a reliable tool for the diagnosis of NASH $[1,5,6]$, and the most sensitive and specific method for providing

\footnotetext{
* Correspondence: onom@kochi-u.ac.jp

${ }^{5}$ Department of Gastroenterology and Hepatology, Kochi Medical School, Kochi, Japan

Full list of author information is available at the end of the article
}

prognostic information. However, it may not be practical to perform liver biopsies in every patient with NAFLD to ascertain the presence of NASH [6]. Moreover, biopsies are associated with significant limitations such as pain, risk of severe complications, sampling errors $[7,8]$, cost, and patient unwillingness to undergo invasive testing. Since it is not easy to distinguish simple steatosis from NASH in diabetes clinics, simple scoring systems to derive progressive NASH are required. Numerous noninvasive panels of tests have been developed to stage liver disease, including a combination of clinical and routine laboratory parameters, as well as specialized tests involving direct markers of fibrosis and elastography [9-20]. Of these, the BAAT (body mass index $[\mathrm{BMI}]$, age, alanine aminotransferase [ALT],
C Biomed Central

두 2012 Sumida et al; licensee BioMed Central Ltd. This is an Open Access article distributed under the terms of the Creative Commons Attribution License (http://creativecommons.org/licenses/by/2.0), which permits unrestricted use, distribution, and reproduction in any medium, provided the original work is properly cited. 
triglycerides) [14], European liver fibrosis (ELF) score [10], Fibrotest (BioPredictive, Paris, France) [9], Fibroscan (Echosens, Paris, France) [12], acoustic radiation force impulse elastography (Mochida Siemens Medical System Co. Ltd., Tokyo, Japan) [15], hyaluronic acid (HA) [16,17], type IV collagen 7S [18], BARD (BMI, aspartate aminotransferase [AST]/ALT ratio [AAR], diabetes mellitus [DM]) [19], N (Nippon) score [20] and the NAFLD fibrosis score (NFS) [21] have been tested in subjects with NAFLD.

The FIB4 index was developed as a noninvasive panel to stage liver disease in subjects with human immunodeficiency virus and hepatitis $\mathrm{C}$ virus (HCV) co-infection [22]. It relies on patient age, AST, ALT, and platelet count, which are routinely measured and are thus available for virtually all subjects with liver disease. This index has also been independently validated in subjects with HCV infection alone [23]. It has recently been demonstrated that its performance characteristics for the diagnosis of advanced fibrosis in NAFLD are better than those of other similar panels that do not require additional testing [24]. However, $74 \%$ of the subjects enrolled in the study were Caucasian, and validation of the FIB4 index in other ethnic groups is required before it can be applied globally. In this study, we therefore aimed to assess the accuracy of the FIB4 index for predicting advanced liver fibrosis in a cohort of Japanese patients with NAFLD.

\section{Methods \\ Patients}

A total of 576 patients with well-characterized and liverbiopsy-confirmed NAFLD between 2002 and 2008 were enrolled from the Japan Study Group of NAFLD (JSGNAFLD), which includes nine hepatology centers in Japan: Center for Digestive and Liver Diseases, Nara City Hospital; Division of Gastroenterology, Yokohama City University Graduate School of Medicine; Department of Medicine and Molecular Science, Graduate School of Biomedical Sciences, Hiroshima University; Department of Gastroenterology and Hepatology, Kochi Medical School; Department of Internal Medicine, Saga Medical School, Saga University; Department of Hepatology, Graduate School of Medicine, Osaka City University; Department of Gastroenterology and Hepatology, Kyoto Prefectural University of Medicine; Division of Gastroenterology and Hematology/Oncology, Department of Medicine, Asahikawa Medical College; and Hepatology Center, Saiseikai Suita Hospital. All patients were also involved in the previous JSG-NAFLD study [25].

The diagnosis of NAFLD was based on the following criteria: (1) liver biopsy showing steatosis in at least 5\% of hepatocytes [26]; and (2) appropriate exclusion of liver diseases of other etiologies, including viral hepatitis, autoimmune hepatitis, drug-induced liver disease, primary biliary cirrhosis, biliary obstruction, hemochromatosis, Wilson's disease, or $\alpha$-1-antitrypsin- deficiencyassociated liver disease. Patients who consumed $>20 \mathrm{~g}$ alcohol per day and patients with evidence of decompensated LC or HCC were excluded. Written informed consent was obtained from all patients at the time of liver biopsy, and the study was conducted in accordance with the Helsinki Declaration [27]. The study protocol was approved by the ethical committee of Nara City Hospital in Nara, Japan.

\section{Anthropometric and laboratory evaluation}

Venous blood samples were taken in the morning after a 12-h overnight fast. Laboratory evaluations in all patients included a blood cell count and measurement of AST, ALT, $\gamma$-glutamyl transpeptidase (GGT), cholinesterase (ChE), total cholesterol, triglyceride, high-density lipoprotein (HDL) cholesterol, albumin, fasting plasma glucose (FPG), immunoreactive insulin (IRI), and ferritin. These parameters were measured using standard clinical chemistry techniques. BMI was also calculated; obesity was defined as BMI $>25$, according to the criteria of the Japan Society for the Study of Obesity [28]. Patients were assigned a diagnosis of DM if they had documented use of oral hypoglycemic medication, a random glucose level $>200 \mathrm{mg} / \mathrm{dL}$, or FPG $>126 \mathrm{mg} / \mathrm{dL}$ [29]. Hypertension was defined as a systolic blood pressure $\geq 130 \mathrm{mmHg}$ or a diastolic blood pressure $\geq 85$ $\mathrm{mmHg}$ or by the use of antihypertensive agents. Dyslipidemia was defined as serum concentrations of triglycerides $\geq 150 \mathrm{mg} / \mathrm{dL}$ or HDL cholesterol $<40 \mathrm{mg} / \mathrm{dL}$ and $<50 \mathrm{mg} / \mathrm{dL}$ for men and women, respectively, or by the use of specific medication [30]. Based on a review of the literature, the following scores were calculated for each patient: FIB4 [22], AAR, AST to platelet ratio index (APRI) [31], age-platelet index (AP index) [32], BARD score [19], N score [20], and NFS [13]. The values for the upper limit of normal were set according to the International Federation of Clinical Chemistry: AST 35 $\mathrm{U} / \mathrm{L}$ for men, $30 \mathrm{U} / \mathrm{L}$ for women, and were comparable to the values used in other analyses. The specific formulae used to determine these scores are shown in Table 1.

\section{Histologic evaluation}

All patients enrolled in this study underwent percutaneous liver biopsy under ultrasonic guidance. The liver specimens were embedded in paraffin and stained with hematoxylin and eosin, and Masson's trichrome. The minimum biopsy size was $20 \mathrm{~mm}$ and the number of portal areas was 10 . The liver biopsy specimens were reviewed by two hepatopathologists (T.O. and Y.S.) who were blinded to the clinical data. Fatty liver was defined 
Table 1 Formulae for determining noninvasive marker panels for detection of liver fibrosis.

\begin{tabular}{|c|c|c|}
\hline Formula & \multicolumn{2}{|l|}{ Equation } \\
\hline FIB4 index & \multicolumn{2}{|c|}{$\left(\right.$ Age $[$ years] $\times$ AST $[I U / L]) /\left(\right.$ platelet count $\left.\left[10^{9} / \mathrm{L}\right] \times \sqrt{\text { ALT[IU } / \mathrm{L}]}\right)$} \\
\hline AST to ALT ratio (AAR) & \multicolumn{2}{|l|}{ AST/ALT } \\
\hline AST to platelet ratio index (APRI) ${ }^{a}$ & \multicolumn{2}{|c|}{$\left(\left[\right.\right.$ AST/ULN]/platelet count $\left.\left[10^{9} / \mathrm{L}\right]\right) \times 100$} \\
\hline \multirow[t]{8}{*}{ Age-platelet index (AP index) } & Age (years) & platelet count $\left(10^{9} / \mathrm{L}\right)$ \\
\hline & $<30=0$ & $<225=0$ \\
\hline & $30-39=1$ & $200-224=1$ \\
\hline & $40-49=2$ & $175-199=2$ \\
\hline & $50-59=3$ & $150-174=3$ \\
\hline & $60-69=4$ & $125-149=4$ \\
\hline & $\geq 70=5$ & $<125=5$ \\
\hline & \multicolumn{2}{|l|}{ Score is the sum of two $(0-10)$} \\
\hline NAFLD fibrosis score & \multicolumn{2}{|c|}{$\begin{array}{l}-1.675+0.037 \times \text { age }(\text { years })+0.094 \times \mathrm{BMl}\left(\mathrm{kg} / \mathrm{m}^{2}\right)+1.13 \times \mathrm{IFG} / \text { diabetes }(\text { yes }=1, \mathrm{no}=0)+0.99 \times \mathrm{AST} / \mathrm{ALT}- \\
0.013 \times \text { platelet count }\left(\times 10^{9} / \mathrm{L}\right)-0.66 \times \text { albumin }(\mathrm{g} / \mathrm{dL}) .\end{array}$} \\
\hline \multirow[t]{4}{*}{ BARD score } & \multicolumn{2}{|c|}{ Scale $0-4$} \\
\hline & \multicolumn{2}{|l|}{$\mathrm{BMI} \geq 28 \mathrm{~kg} / \mathrm{m}^{2}=1$ point } \\
\hline & \multicolumn{2}{|l|}{ AST $/ A L T \geq 0.8=2$ points } \\
\hline & \multicolumn{2}{|l|}{ Diabetes $=1$ point } \\
\hline \multirow[t]{5}{*}{ N (Nippon) score } & \multicolumn{2}{|l|}{ Scale $0-4$} \\
\hline & \multicolumn{2}{|l|}{ female sex $=1$ point } \\
\hline & \multicolumn{2}{|l|}{ older age $(>60$ years $)=1$ point } \\
\hline & \multicolumn{2}{|l|}{ type 2 diabetes $=1$ point } \\
\hline & \multicolumn{2}{|l|}{ hypertension $=1$ point } \\
\hline
\end{tabular}

BMI, body mass index; IFG, impaired fasting glucose; INR, international normalized ratio; ULN, upper limit of normal. ${ }^{a}$ ULN for AST: 30 in women, 35 in men.

as the presence of steatosis in at least $5 \%$ hepatocytes, while steatohepatitis was diagnosed by steatosis, inflammation, and hepatocyte ballooning $[2,3,26]$. The individual parameters of NASH histology, including fibrosis, were scored independently using the NASH Clinical Research Network (CRN) scoring system developed by the NASH CRN [26]. Advanced fibrosis was classified as stage 3 or 4 disease (bridging fibrosis or cirrhosis).

\section{Statistical analysis}

Statistical analysis was conducted using SPSS 19.0 software (SPSS, Inc., Chicago, IL). Continuous variables were expressed as mean \pm standard deviation (SD), or median (interquartile range). Qualitative data were presented as numbers with percentages in parentheses. Statistical differences in quantitative data were determined using the $t$ test or Mann-Whitney U test. Fisher's exact probability test or $\chi^{2}$ analysis was used for qualitative data (Table 2). The sensitivity and specificity for each value of each test were calculated to assess the accuracy of the clinical scoring system in differentiating between advanced and mild fibrosis, and receiver operating characteristic (ROC) curves were constructed by plotting the sensitivity against (1 - specificity) at each value (Figure 1 ). The diagnostic performances of the scoring systems were assessed by analysis of ROC curves. The most commonly used index of accuracy was the area under the ROC curve (AUROC), with values close to 1.0 indicating high diagnostic accuracy. (Table 3). The sensitivity, specificity, positive predictive value (PPV), and negative predictive value (NPV) were calculated for the two cut-off values $(<1.45$ and $>3.25)$ proposed by Sterling [22] and those $(<1.30$ and $>2.67)$ proposed by Shah [24]. Differences were considered statistically significant at $p<0.05$.

\section{Results}

A total of 576 subjects were included in this analysis. Of these, 280 (49\%) were women and 418 (73\%) were obese (Table 2); 241 (42\%) had type 2 DM and 184 (32\%) were hypertensive. A total of 319 subjects had steatohepatitis, of whom 64 subjects had advanced fibrosis. As expected, subjects with more advanced fibrosis were significantly older, predominantly female, and more likely to be hypertensive, to have type $2 \mathrm{DM}$, to have higher AST, AAR, GGT, FPG, and IRI, and to have lower hemoglobin, platelet count, albumin, ChE, total cholesterol, and triglyceride. Regarding the individual components of the FIB4 score, the mean $( \pm$ SD) or median [interquartile range] values were as follows: age (52.3 \pm 15.4 years); AST (43 [30-67] IU/L); ALT (69 [43-112] $\mathrm{IU} / \mathrm{L})$, and platelets $\left(227 \pm 67 \times 10^{9} / \mathrm{L}\right)($ Table 2$)$. The distribution of fibrosis stages included stage $0(n=263)$, stage $1(n=169)$, stage $2(n=80)$, stage $3(n=45)$, and 
Table 2 Characteristics of study population and values of noninvasive fibrosis marker panels ${ }^{\mathrm{a}}$.

\begin{tabular}{|c|c|c|c|c|}
\hline & $\begin{array}{l}\text { Total } \\
(n=576)\end{array}$ & Fibrosis stage $0-2(n=512)$ & Fibrosis stage $3-4(n=64)$ & $p$-value ${ }^{b}$ \\
\hline Age $(y r)$ & $52.3 \pm 15.4$ & $51.2 \pm 15.5$ & $62.0 \pm 10.1$ & $<0.0001$ \\
\hline Gender (female) & $280(49 \%)$ & $235(46 \%)$ & $45(70 \%)$ & 0.0003 \\
\hline BMI $\left(\mathrm{kg} / \mathrm{m}^{2}\right)$ & $27.9 \pm 4.9$ & $27.8 \pm 4.9$ & $28.6 \pm 4.8$ & 0.2138 \\
\hline Obesity (BMI > 25) & $418(73 \%)$ & $369(72 \%)$ & $49(77 \%)$ & 0.5524 \\
\hline Hypertension (yes) & $184(32 \%)$ & $150(29 \%)$ & $34(53 \%)$ & 0.0062 \\
\hline Type 2 diabetes (yes) & $241(42 \%)$ & $199(39 \%)$ & $42(66 \%)$ & 0.0001 \\
\hline Hemoglobin (g/dL) & $14.6 \pm 2.0$ & $14.7 \pm 2.0$ & $13.7 \pm 2.0$ & 0.0001 \\
\hline Platelet count $\left(\times 10^{9} / \mathrm{L}\right)$ & $227 \pm 67$ & $235 \pm 64$ & $162 \pm 52$ & $<0.0001$ \\
\hline AST (IU/L) & $43(30-67)$ & $41(29-64)$ & $61(47-77)$ & $<0.0001$ \\
\hline ALT (IU/L) & $69(43-112)$ & $69(43-69)$ & $62(46-94)$ & 0.5074 \\
\hline AST/ALT ratio & $0.65(0.52-0.82)$ & $0.63(0.51-0.78)$ & $0.98(0.73-1.21)$ & $<0.0001$ \\
\hline GGT (IU/L) & $\begin{array}{l}60(39-99) \\
(n=572)\end{array}$ & $\begin{array}{l}57(36-92) \\
(n=508)\end{array}$ & $84(59-128)$ & $<0.0001$ \\
\hline Albumin $(\mathrm{g} / \mathrm{dL})$ & $4.4 \pm 0.4$ & $4.4 \pm 0.4$ & $4.1 \pm 0.4$ & $<0.0001$ \\
\hline Cholinesterase (IU/L) & $\begin{array}{l}380(330-433) \\
(n=527)\end{array}$ & $\begin{array}{l}385(337-439) \\
(\mathrm{n}=466)\end{array}$ & $\begin{array}{l}297(244-367) \\
(n=61)\end{array}$ & $<0.0001$ \\
\hline Total cholesterol (mg/dL) & $\begin{array}{l}209 \pm 40 \\
(n=467)\end{array}$ & $\begin{array}{l}210 \pm 39 \\
(n=409)\end{array}$ & $\begin{array}{l}198 \pm 42 \\
(n=58)\end{array}$ & 0.0484 \\
\hline Triglyceride (mg/dL) & $\begin{array}{l}147(107-207) \\
(\mathrm{n}=566)\end{array}$ & $\begin{array}{l}150(109-212) \\
(n=502)\end{array}$ & $\begin{array}{l}131(95-184) \\
(n=64)\end{array}$ & 0.0364 \\
\hline HDL-C (mg/dL) & $\begin{array}{l}50 \pm 17 \\
(n=548)\end{array}$ & $\begin{array}{l}50 \pm 17 \\
(n=487)\end{array}$ & $\begin{array}{l}51 \pm 13 \\
(n=61)\end{array}$ & 0.7516 \\
\hline LDL-C (mg/dL) & $\begin{array}{l}128 \pm 33 \\
(n=405)\end{array}$ & $\begin{array}{l}129 \pm 32 \\
(n=363)\end{array}$ & $\begin{array}{l}120 \pm 42 \\
(n=42)\end{array}$ & 0.1666 \\
\hline Ferritin $(\mathrm{ng} / \mathrm{mL})$ & $173(92-300)$ & $169(91-292)$ & $216(128-349)$ & 0.0627 \\
\hline FPG (mg/dL) & $\begin{array}{l}103(94-122) \\
(n=524)\end{array}$ & $\begin{array}{l}103(94-119) \\
(n=462)\end{array}$ & $\begin{array}{l}111(95-138) \\
(n=62)\end{array}$ & 0.0166 \\
\hline IRI $(\mu \mathrm{U} / \mathrm{mL})$ & $11.6(7.8-18.4)$ & $11.3(7.5-17.4)$ & $17.3(11.3-26.2)$ & $<0.0001$ \\
\hline FIB4 index & $1.23(0.77-2.02)$ & $1.13(0.71-1.79)$ & $3.17(1.88-4.25)$ & $<0.0001$ \\
\hline $\begin{array}{l}\text { AST/ALT ratio } \\
\text { (AAR) }\end{array}$ & $0.65(0.52-0.82)$ & $0.63(0.51-0.78)$ & $0.98(0.73-1.21)$ & $<0.0001$ \\
\hline AST to platelet ratio index (APRI) & $0.61(0.40-0.98)$ & $0.57(0.38-0.92)$ & $1.22(0.86-1.79)$ & $<0.0001$ \\
\hline Age-platelet index (AP index) & $4(2-6)$ & $3(2-5)$ & $7(5-8)$ & $<0.0001$ \\
\hline NAFLD fibrosis score & $\begin{array}{l}-1.82 \\
(-3.04 \text { to }-0.58)\end{array}$ & $\begin{array}{l}-2.07 \\
(-3.25 \text { to }-0.95)\end{array}$ & $\begin{array}{l}0.25 \\
(-0.60-1.06)\end{array}$ & $<0.0001$ \\
\hline BARD score & & & & $<0.0001$ \\
\hline 0 & $144(25 \%)$ & $138(27 \%)$ & $6(9 \%)$ & \\
\hline 1 & $201(35 \%)$ & $194(38 \%)$ & $7(11 \%)$ & \\
\hline 2 & $112(19 \%)$ & 99 (19\%) & $13(20 \%)$ & \\
\hline 3 & $88(15 \%)$ & $62(12 \%)$ & $26(41 \%)$ & \\
\hline 4 & $31(5 \%)$ & $19(4 \%)$ & $12(19 \%)$ & \\
\hline N score & & & & $<0.0001$ \\
\hline 0 & 135 (23\%) & $135(26 \%)$ & $0(0 \%)$ & \\
\hline 1 & $170(30 \%)$ & 157 (31\%) & $13(20 \%)$ & \\
\hline 2 & 118 (20\%) & 96 (19\%) & $22(34 \%)$ & \\
\hline 3 & 99 (17\%) & $82(16 \%)$ & $17(27 \%)$ & \\
\hline 4 & $54(9 \%)$ & $42(8 \%)$ & $12(19 \%)$ & \\
\hline
\end{tabular}

BMI, body mass index; AST, aspartate aminotransferase; ALT, alanine aminotransferase; GGT, $\gamma$-glutamyl transpeptidase; HDL, high-density lipoprotein; LDL, lowdensity lipoprotein; FPG, fasting plasma glucose; and IRI, immuno-reactive insulin. $a$ Values are mean \pm SD, median (interquartile range), counts (\%), as appropriate. $b$ Values from univariate ordinal logistic regression, Mann-Whitney, or $\chi^{2}$ analysis, as appropriate. 


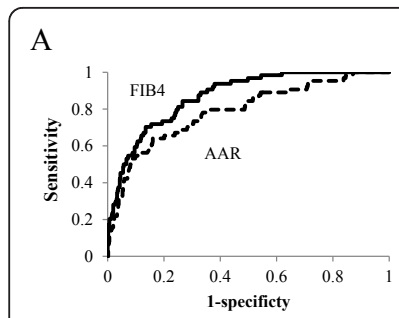

$\mathrm{C}$

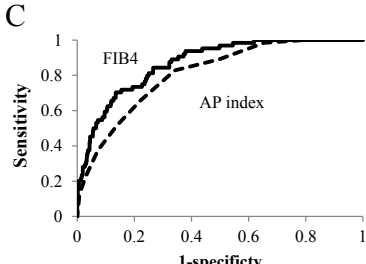

$\mathrm{E}$
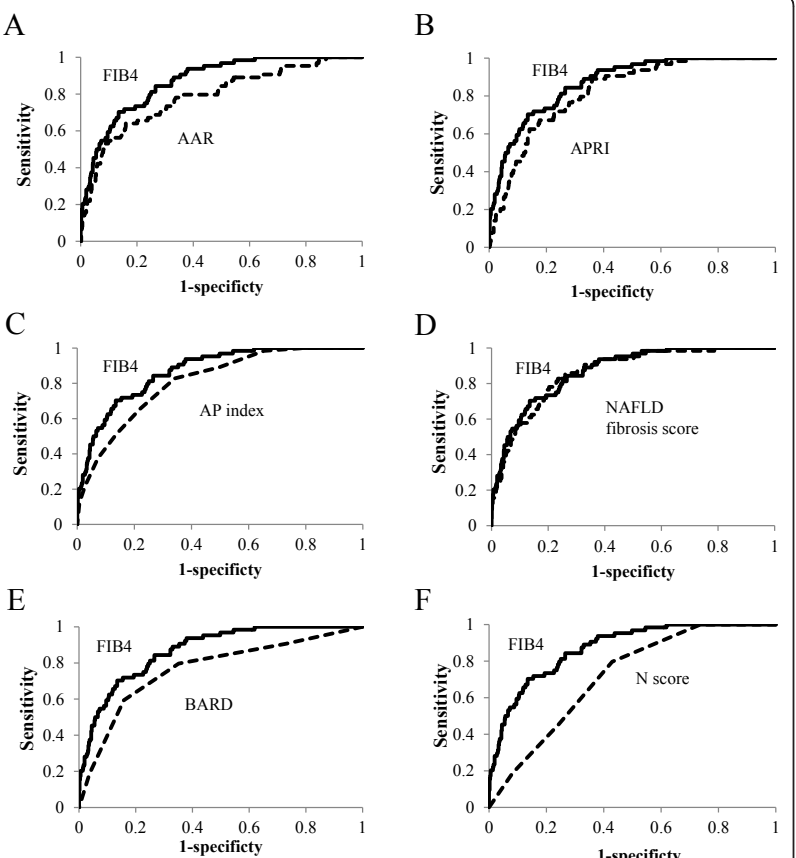

$\mathrm{D}$

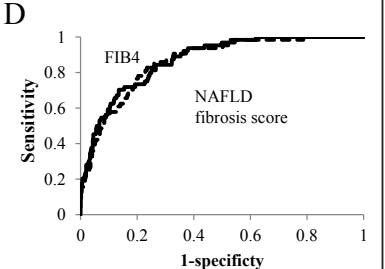

F

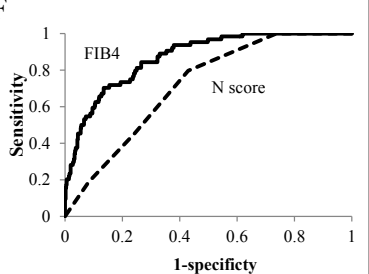

Figure 1 Comparison of ROCs of FIB4 and other panels for the diagnosis of advanced fibrosis.

stage $4(n=19)$. FIB4 values for the whole sample ranged from 0.17-10.74. The median FIB4 score was 1.23 (interquartile range, 0.77-2.02) (Table 3). The mean (interquartile range) FIB4 indices for stages 0, 1, 2, 3, and 4 were 1.09 (0.61-1.34), 1.40 (0.77-1.88), 2.36 (1.44$3.15), 3.23$ (1.82-4.04), and 4.48 (3.19-5.17), respectively $(p<0.0001$ by analysis of variance). The mean (interquartile range) FIB4 index was 1.13 (0.71-1.79) in patients with stage $0-2$ fibrosis and 3.17 (1.88-4.25) in patients with stage $3-4$ fibrosis $(p<0.0001)$ (Table 2).

The sensitivity and specificity of FIB4 along the ROC were assessed first. At a sensitivity of $90 \%($ FIB4 $=1.45$ ) the specificity was $35 \%$, while at a specificity of $90 \%$ (FIB4 $=2.67$ ), the sensitivity was $52 \%$. ROC curves were then developed for each of the noninvasive marker panels and superimposed, to determine which score would have the most clinical utility (Figure 1). ROC curves were created to determine the utility of the indices for predicting advanced fibrosis (stage 3 and 4 versus lower scores). The AUROC was greatest for FIB4 (0.871), followed by NFS (0.863), APRI (0.823), AP index (0.810), AAR (0.788), BARD score (0.765), and $\mathrm{N}$ score (0.715) (Table 3). As the NPVs for FIB4 index, AAR, APRI, AP index, NFS, BARD score, and N score were all greater than $95 \%$ using their lower cut-offs, these tests may have sufficient accuracy to be used clinically to exclude advanced fibrosis. Using this approach, a significant proportion of patients could avoid liver biopsy using each of these tests (Table 3). As the PPV were modest for all noninvasive tests, ranging from 19\% to $53 \%$, it was felt they were not accurate enough to be used as an alternative to liver biopsy. The PPV for FIB4 is highest among other noninvasive tests.

Using the low cut-off point proposed by Sterling and colleagues $(<1.45)$ [22], 330 of $336(98.3 \%)$ patients without stage 3 or 4 fibrosis were correctly staged, while only $6(1.7 \%)$ were under-staged (Table 4$)$. All of the 6 patients with advanced fibrosis but FIB4 index below the low cut-off point had stage 3 fibrosis, none had stage 4 fibrosis. The NPV of this cut-off for stage 3 or 4 fibrosis was $98 \%$. Using the high cut-off point proposed by Sterling and colleagues (> 3.25) [24], 31 of 59 (52.5\%) patients with stage 3 or 4 fibrosis were correctly staged, while $28(47.5 \%)$ were over-staged. Among the 28 patients without advanced fibrosis but FIB4 index above the high cut-off point, 18 had stage 2 fibrosis, 6 had stage 1 , and 4 had no fibrosis. The PPV of this cut-off for stage 3 or 4 fibrosis was $53 \%$. A total of 395 patients (69\% of the cohort) had a FIB4 index $<1.45$ or $>3.25$; FIB4 identified the absence or presence of advanced fibrosis with $91 \%$ accuracy in these 361 subjects. A total of 181 subjects (31\%) had FIB4 values in the indeterminate range (1.4-3.25).

Table 3 Accuracy of noninvasive fibrosis marker panels.

\begin{tabular}{|c|c|c|c|c|c|c|}
\hline Fibrosis panel & AUROC & Cut-off values & $\begin{array}{l}\text { Sensitivity } \\
\text { (\%) }\end{array}$ & $\begin{array}{l}\text { Specificity } \\
\text { (\%) }\end{array}$ & $\begin{array}{l}\text { PPV } \\
\text { (\%) }\end{array}$ & $\begin{array}{l}\text { NPV } \\
\text { (\%) }\end{array}$ \\
\hline FIB4 index & 0.871 & $\begin{array}{l}1.45 \\
3.25\end{array}$ & $\begin{array}{l}90 \\
48\end{array}$ & $\begin{array}{l}64 \\
95\end{array}$ & $\begin{array}{l}24 \\
53\end{array}$ & $\begin{array}{l}98 \\
94\end{array}$ \\
\hline AST/ALT ratio (AAR) & 0.788 & $\begin{array}{l}0.8 \\
1\end{array}$ & $\begin{array}{l}66 \\
48\end{array}$ & $\begin{array}{l}76 \\
92\end{array}$ & $\begin{array}{l}26 \\
44\end{array}$ & $\begin{array}{l}95 \\
94\end{array}$ \\
\hline AST to platelet ratio index (APRI) & 0.823 & 1 & 67 & 81 & 31 & 95 \\
\hline Age-platelet index (AP index) & 0.810 & 6 & 66 & 78 & 27 & 95 \\
\hline NAFLD fibrosis score & 0.863 & $\begin{array}{l}-1.455 \\
0.676\end{array}$ & $\begin{array}{l}92 \\
33\end{array}$ & $\begin{array}{l}63 \\
96\end{array}$ & $\begin{array}{l}24 \\
50\end{array}$ & $\begin{array}{l}98 \\
92\end{array}$ \\
\hline BARD score & 0.765 & 2 & 80 & 65 & 22 & 97 \\
\hline N score & 0.715 & 2 & 80 & 58 & 19 & 96 \\
\hline
\end{tabular}

AUROC, area under the receiver operating characteristics curve; PPV, positive predictive value; NPV, negative predictive value 
Table 4 Proportion of patients who may potentially avoid liver biopsy using the simple non-invasive tests to exclude advanced fibrosis.

\begin{tabular}{llll}
\hline Fibrosis panel & Cut-off values & Patients avoiding liver biopsy $^{a}$ & False negative result $^{\circ}$ \\
\hline FIB4 index & $<1.45$ & $336 / 576(58 \%)$ & $6(2 \%)$ \\
& $<1.30$ & $308 / 576(53 \%)$ & $4(1 \%)$ \\
AST/ALT ratio (AAR) & $<0.8$ & $413 / 576(72 \%)$ & $22(5 \%)$ \\
AST to platelet ratio index (APRI) & $<1$ & $435 / 576(76 \%)$ & $21(5 \%)$ \\
Age-platelet index (AP index) & $<6$ & $421 / 576(73 \%)$ & $22(5 \%)$ \\
NAFLD fibrosis score & $<-1.455$ & $328 / 576(57 \%)$ & $5(2 \%)$ \\
BARD score & $<2$ & $355 / 576(62 \%)$ & $13(4 \%)$ \\
N score & $<2$ & $305 / 576(53 \%)$ & $13(4 \%)$ \\
\hline
\end{tabular}

${ }^{a}$ Patients with a value below the cut-off.

On the other hand, using the low cut-off point proposed by Shah and colleagues $(<1.30)$ [24], 304 of 308 (99\%) patients without stage 3 or 4 fibrosis were correctly staged, while only $4(1 \%)$ were under-staged (Table 4). All of the 4 patients with advanced fibrosis but FIB4 index below the low cut-off point had stage 3 fibrosis and none had stage 4 fibrosis. The NPV of this cut-off for stage 3 or 4 fibrosis was $99 \%$. Using the high cut-off point proposed by Shah and colleagues $(>2.67)$, 38 of $89(43 \%)$ patients with stage 3 or 4 fibrosis were correctly staged, while 51 (57\%) were over-staged. Among the 51 patients without advanced fibrosis but NAFLD fibrosis scores above the high cut-off point, 28 had stage 2 fibrosis, 14 had stage 1 , and 9 had no fibrosis. The PPV of this cut-off for stage 3 or 4 fibrosis was $43 \%$. A total of 397 patients ( $69 \%$ of the cohort) had a FIB4 index $<1.30$ or $>2.67$; FIB4 identified the absence or presence of advanced fibrosis with $86 \%$ accuracy in these 342 subjects. A total of 179 subjects (31\%) had FIB4 values in the indeterminate range (1.30-2.67). Thus the prevalence of patients in the indeterminate range was similar using the two different cut-off values, but the number of patients with true positive or true negative predictions (accuracy) was higher using Sterling et al.'s cut-off values compared with Shah et al.'s (361 patients versus 342 patients). If liver biopsies were only performed in patients with an FIB4 index above the low cut-off point (>1.45) proposed by Sterling, $336(58 \%)$ of 576 biopsies could be avoided (Table 4).

The diagnostic accuracy of FIB4 index for detecting advanced fibrosis (stage 3-4) was also compared to that of NFS (Table 5). Three hundred and seventy patients (64\% of the cohort) had an NFS $<-1.455$ or $>0.676$; NFS identified the absence or presence of advanced fibrosis with $93 \%$ accuracy in these 344 subjects. A total of 206 subjects (36\%) had NFS values in the indeterminate range (-1.455-0.676). Although the accuracy of NFS was higher $(93 \%)$ than that of FIB4 $(86 \%)$, more patients were correctly staged with FIB4 $(n=361)$ than with NFS $(n=344)$. Moreover, the percentage of patients in the undetermined range was lower for the FIB4 index (31\%) than for NFS (36\%). Using the cut-off values reported by Sterling and colleagues, discrepancies between FIB4 index and NFS were observed in 146 (39\%) patients (Table 5). Patients were categorized into three groups, "low-risk" (< 10\%), "intermediate-risk" (10$30 \%$ ) and "high-risk" (> 30\%), based on the combination of FIB4 index and NFS (Table 5). Only 1 patient (0.4\%)

Table 5 Categorized risk groups for advanced fibrosis according to combined FIB4 index and NAFLD fibrosis score (NFS).

\begin{tabular}{|c|c|c|c|c|c|}
\hline & & \multicolumn{3}{|c|}{$\begin{array}{l}\text { FIB4 index } \\
\text { (cut-off values proposed by Sterling et al.) }\end{array}$} & \multirow[t]{2}{*}{ Total } \\
\hline & & Low cut-off point $(<1.45)$ & $\begin{array}{l}\text { Indeterminate } \\
(1.45-3.25)\end{array}$ & High cut-off point (> 3.25) & \\
\hline \multirow[t]{3}{*}{ NFS } & Low cut-off point $(<-1.455)$ & $\begin{array}{l}283 \\
{[1(0.4 \%)]^{a}}\end{array}$ & $\begin{array}{l}42 \\
{[4(9.5 \%)]^{a}}\end{array}$ & $\begin{array}{l}3 \\
{[0(0.0 \%)]^{\text {a }}}\end{array}$ & $\begin{array}{l}328(56.9 \%) \\
{[5(1.5 \%)]}\end{array}$ \\
\hline & Indeterminate $(-1.455-0.676)$ & $\begin{array}{l}53 \\
{[5(9.4 \%)]^{a}}\end{array}$ & $\begin{array}{l}122 \\
{[19(15.6 \%)]^{b}}\end{array}$ & $\begin{array}{l}31 \\
{[14(45.2 \%)]^{c}}\end{array}$ & $\begin{array}{l}206(35.8 \%) \\
{[38(18.4 \%)]}\end{array}$ \\
\hline & High cut-off point $(>0.676)$ & 0 & $\begin{array}{l}17 \\
{[4(23.5 \%)]^{b}}\end{array}$ & $\begin{array}{l}25 \\
{[17(68.0 \%)]^{c}}\end{array}$ & $\begin{array}{l}42(7.3 \%) \\
{[21(50.0 \%)]}\end{array}$ \\
\hline Total & & $\begin{array}{l}336(58.3 \%) \\
{[6(1.7 \%)]}\end{array}$ & $\begin{array}{l}181(31.4 \%) \\
{[27(14.9 \%)]}\end{array}$ & $\begin{array}{l}59(10.2 \%) \\
{[31(52.5 \%)\}}\end{array}$ & $\begin{array}{l}576(100 \%) \\
{[64(11.1 \%)]}\end{array}$ \\
\hline
\end{tabular}

Total number of patients [stage 3-4 (\%)]

Patients were categorized into three groups, "low-risk" $(<10 \%)$ a " "intermediate-risk" (10-30\%) ${ }^{\text {b }}$ and "high-risk" (> 30\%) ${ }^{\mathrm{c}}$, based on the combination of FIB4 index and NFS. 
of 243 patients with the low cut-off points for both FIB4 index and NFS had advanced fibrosis.

\section{Discussion}

The AUROC of FIB4 was 0.871 for the diagnosis of advanced fibrosis, which was superior to those of the other noninvasive panels tested. For a value $<1.45$, fibrosis could be excluded with $98 \%$ certainty (NPV 98\%) whereas for a value $>3.25$, the presence of significant fibrosis could be predicted with 53\%. Despite the limited sensitivity of the FIB4 index in a population with a low prevalence of advanced fibrosis, the score was useful for ruling out advanced fibrosis. In our cohort, 58\% of the liver biopsies could have been avoided if the procedure was not performed in patients with a FIB4 index below the low cut-off point $(<1.45)$. The score would therefore be particularly useful for reducing the number of unnecessary liver biopsies performed, and thus the costs of managing NAFLD patients in Asia, where advanced fibrosis is uncommon. A high cut-off FIB4 index of 2.67 which has been proposed by Shah and colleagues [24] had a low PPV (43\%) in predicting stage 3 or 4 fibrosis. Our results contrast with those reported by Shah and colleagues [24], where a high cut-off FIB4 index of 2.67 had an $80 \%$ PPV in predicting stage 3 or 4 fibrosis; however the prevalence of advanced fibrosis in our study was only $11 \%$, compared to $23 \%$ in Shah et al.'s study. Our study was therefore unable to reliably validate the high cut-off point, and larger Asian studies are warranted to investigate this. The FIB4 index was higher in our population than in Shah et al.'s study; stage $0-2: 1.13(0.71-1.79)$ versus 0.97 (0.68-1.37), stage 3-4: 3.17 (1.88-4.25) versus 1.98 (1.28-3.08), probably because of older age, higher levels of ALT, and lower levels of platelets in our population.

The BARD score developed by Harrison et al. represents the weighted sum of three easily available variables (BMI $\geq 28 \mathrm{~kg} / \mathrm{m}^{2}$ [1 point], AAR $\geq 0.8$ [2 points], and DM [1 point $]$ ), and the authors demonstrated that a score of 2-4 was associated with an odds ratio of 17 for predicting advanced fibrosis [19]. Although BARD score is simple to calculate, our validation study failed to detect any advantage of this score over FIB4; a BARD score of $\geq 2$ was associated with a sensitivity, specificity, PPV and NPV for detecting advanced fibrosis of 80, 65, 22 and $97 \%$, respectively. Consistent with the present study, Fujii and colleagues reported significantly poorer applicability of BARD in Japanese patients with NAFLD, compared with Caucasian subjects [33]. It has been suggested that BARD score is less predictive of advanced fibrosis in Japanese NAFLD patients because they are less obese than those in western countries. The $\mathrm{N}$ score (the total number of the following risk factors: female sex, age > 60 years, type $2 \mathrm{DM}$, and hypertension), which was established on the basis of data from 182 Japanese NAFLD patients in multiple centers in Nagasaki [20], requires no detailed laboratory measurements, but was not found to be superior to FIB4 index in our validation study. Angulo et al. found that the NFS, which consists of six variables (age, BMI, AAR, IFG/ DM, platelet count, and albumin), reliably predicted advanced fibrosis in NAFLD patients [21]. In 428 (74\%) of the subjects in the present study, FIB4 index was in accordance with NFS. The combination of two scoring systems could help to identify patients likely to have advanced fibrosis. Patients with FIB4 values above the high cut-off point $(>3.25)$ and NFS values above the low cut-off point $(>-1.455)$ were at high risk $(>30 \%)$ for advanced fibrosis. If both FIB4 and NFS were applied to Japanese patients with NAFLD, patients with either FIB4 or NFS values below the low cut-off points (376/576, 65.3\%) could avoid liver biopsies. In this way, when FIB4 was combined with NFS, its ability to predict or exclude advanced fibrosis improved further. In summary, the current study demonstrated that the FIB4 index, which can be established using a simple, relatively inexpensive method, correlated with the stage of fibrosis in adult subjects with NAFLD.

Type IV collagen is one of extracellular matrices that are produced by hepatic fibroblasts. The $7 \mathrm{~S}$ domain in the $\mathrm{N}$-terminus of type IV collagen is inserted in tissues and released into the blood by turnover in connective tissues. Therefore, the serum 7S domain level increases in parallel with the amount of fibrosis and in synthesis from stellate cells and myofibroblasts following increased liver fibrosis. In Japan, type IV collagen 7S is now widely used for assessing the extent of hepatic fibrosis in chronic liver diseases. Our data demonstrated that a cutoff point of $5.4 \mathrm{ng} / \mathrm{ml}$ provided a sensitivity and specificity of $86 \%$ and $87 \%$, respectively, to detect advanced stage of NASH. The AUROC of type IV collagen $7 \mathrm{~s}$ was: 0.926 for the diagnosis of advanced fibrosis, which was superior to FIB4 (data not shown). This data suggest that type IV collagen $7 \mathrm{~S}$ is one of the best parameters among non-invasive parameters, but it costs too much to be determined routinely.

On the other hand, hepatic steatosis is frequently found in patients with HCV infection. Therefore, we also evaluated the value of FIB4 index in $185 \mathrm{HCV}$ infected patients with hepatic steatosis, including those with 72 advanced and 113 mild fibrosis. The AUROC of FIB4 was 0.808 for the diagnosis of advanced fibrosis. For a value $<1.45$, fibrosis could be excluded with $89 \%$ certainty (NPV 89\%) whereas for a value $>3.25$, the presence of advanced fibrosis could be predicted with $82 \%$ (data not shown).

This study had several limitations. First, the proportion of subjects with advanced fibrosis was small, as 
reported in other Asian studies [34], and further Asian studies with more patients with advanced fibrosis are warranted. Second, patients were recruited from hepatology centers in Japan with a particular interest in studying NAFLD, and the possibility of some referral bias could therefore not be ruled out. Patient selection bias could also have existed, because liver biopsy might have been considered for NAFLD patients who were likely to have NASH. The findings may thus not represent NAFLD patients in the wider community. However, this would introduce a negative bias, as NAFLD patients in the community would be likely to have milder liver disease, thus increasing the NPV of the FIB4 index. We also acknowledge that pathologic diagnosis was mainly determined using liver tissues derived from percutaneous liver biopsies, which are prone to sampling errors or interobserver variability $[7,8]$. As recent studies suggest that low normal ALT value does not guarantee freedom from underlying NASH with advanced fibrosis [35-37], it remains to be solved whether FIB4 index can be useful for predicting advanced fibrosis in NAFLD subjects with normal ALT. According to our preliminary data by JSG-NAFLD, the AUROC of FIB4 was 0.810 for the diagnosis of advanced fibrosis in 187 biopsy-proven NAFLD patients with normal ALT levels (data not shown). Our data support the hypothesis that FIB4 index could also be used in the Japanese NAFLD population with normal ALT.

\section{Conclusion}

The FIB4 index demonstrated a good NPV for excluding advanced fibrosis in Japanese NAFLD patients, and could thus be used to reduce the burden of liver biopsies. Larger Asian studies are required to validate the high cut-off point of the FIB4 index. However, the FIB4 test also has several serious limitations, in common with other noninvasive tests for fibrosis, and further research is needed before simple noninvasive tests, including the FIB4 test, can replace liver biopsies in the vast majority of patients.

\section{Abbreviations \\ AAR: AST/ALT ratio; AST: aspartate aminotransferase; ALT: alanine aminotransferase; AP index: age to platelet index; APRI- aspartate aminotransferase to platelet ratio index; AUROC: area under the receiver operating characteristic; NAFLD: nonalcoholic fatty liver disease; NASH: nonalcoholic steatohepatitis; NFS: NAFLD fibrosis score; NPV: negative predictive value; PPV: positive predictive value.}

\section{Acknowledgments}

This study was supported by a Grant from the Chiyoda Mutual Life Foundation. The authors thank the following individuals for assistance in preparation of this manuscript: Kyoko Sakai, M.D.,Ph.D., Tasuku Hara, M.D., Center for Digestive and Liver Diseases, Nara City Hospital; Yuki Kimura, M.D., Tomokazu Ishitobi, M.D., Department of Medicine and Molecular Science, Graduate School of Biomedical Sciences, Hiroshima University; Takaaki Ohtake, M.D., Ph.D., Division of Gastroenterology and Hematology/Oncology,
Department of Medicine, Asahikawa; Yutaka Inada, M.D., Naohisa Yoshida M. D., Ph.D., Kanji Yamaguchi, M.D., Ph.D., Kohichiroh Yasui, M.D., Ph.D., Department of Gastroenterology and Hepatology, Kyoto Prefectural University of Medicine; Yoshihiro Kamada, M.D., Ph.D., Department of Gastroenterology and Hepatology, Osaka University Graduate School of Medicine; Atsushi Nakajima, M.D., Ph.D., Division of Gastroenterology, Yokohama City University Graduate School of Medicine.

\section{Author details}

${ }^{1}$ Center for Digestive and Liver Diseases, Nara City Hospital, Nara, Japan. Division of Gastroenterology, Yokohama City University Graduate School of Medicine, Yokohama, Japan. ${ }^{3}$ Department of Medicine and Molecular Science, Graduate School of Biomedical Sciences, Hiroshima University, Hiroshima, Japan. ${ }^{4}$ Department of Gastroenterology and Hepatology, Kyoto Prefectural University of Medicine, Kyoto, Japan. ${ }^{5}$ Department of Gastroenterology and Hepatology, Kochi Medical School, Kochi, Japan. ${ }^{6}$ Department of Hepatology, Graduate School of Medicine, Osaka City University, Osaka, Japan. ${ }^{7}$ Department of Internal Medicine, Saga Medical School, Saga University, Saga, Japan. ${ }^{8}$ Division of Gastroenterology and Hematology/Oncology, Department of Medicine, Asahikawa Medical College, Asahikawa, Japan. ${ }^{9}$ School of Biomedical Informatics, University of Texas Health Science Center at Houston, Houston, TX, USA. ${ }^{10}$ Hepatology Center, Saiseikai Suita Hospital, Suita, Japan.

\section{Authors' contributions}

YS: study concept and design, drafting of the manuscript, MY: acquisition of data, $\mathrm{HH}$ : acquisition of data, Yl: critical revision of the manuscript for important intellectual content. MO: study concept and design, HF: acquisition of data, YE: acquisition of data, YS: acquisition of data, NA: statistical analysis, KK: critical revision of the manuscript for important intellectual content, KF: acquisition of data, KC: critical revision of the manuscript for important intellectual content TS: critical revision of the manuscript for important intellectual content NK: critical revision of the manuscript for important intellectual content KF: critical revision of the manuscript for important intellectual content YK: critical revision of the manuscript for important intellectual content, TY: critical revision of the manuscript for important intellectual content, TO: study supervision, JSGNAFLD: acquisition of data, study supervision. All authors read and approved the final manuscript.

\section{Competing interests}

The authors declare that there is no duality of interest associated with this manuscript.

Received: 22 April 2011 Accepted: 5 January 2012

Published: 5 January 2012

\section{References}

1. Ludwig J, Viggiano TR, McGill DB, Ott BJ: Non-alcoholic steatohepatitis. Mayo Clinic experiences with a hitherto unnamed disease. Mayo Clin Proc 1980, 55:434-438.

2. Matteoni CA, Younossi ZM, Gramlich T, Boparai N, Liu YC, McCullough AJ: Nonalcoholic fatty liver diseases: a spectrum of clinical and pathological severity. Gastroenterology 1999, 116:1413-1419.

3. Rafiq N, Bai C, Fang Y, Srishord M, McCullough A, Gramlich T, Younossi ZM: Long-term follow-up of patients with nonalcoholic fatty liver. Clin Gastroenterol Hepatol 2009, 7:234-238.

4. Ono M, Saibara T: Clinical features of nonalcoholic steatohepatitis in Japan: evidence from literature. J Gastroentero/ 2006, 41:725-732.

5. Vuppalanchi R, Chalasani N: Nonalcoholic fatty liver disease and nonalcoholic steatohepatitis: Selected practical issues in their evaluation and management. Hepatology 2009, 49:306-317.

6. Wieckowska A, McCullough AJ, Feldstein AE: Noninvasive diagnosis and monitoring of nonalcoholic steatohepatitis: present and future. Hepatology 2007, 46:582-589.

7. Ratziu V, Charlotte F, Heurtier A, Gombert S, Giral P, Bruckert E, Grimaldi A, Capron F, Poynard T, LIDO Study Group: Sampling variability of liver biopsy in nonalcoholic fatty liver disease. Gastroenterology 2005, 128:1898-1906.

8. Merriman RB, Ferrell LD, Patti MG, Weston SR, Pabst MS, Aouizerat BE, Bass NM: Correlation of paired liver biopsies in morbidly obese patients 
with suspected nonalcoholic fatty liver disease. Hepatology 2006, 44:874-880.

9. Ratziu V, Massard J, Charlotte F, Messous D, Imbert-Bismut F, Bonyhay L, Tahiri M, Munteanu M, Thabut D, Cadranel JF, Le Bail B, de Ledinghen V Poynard T, LIDO Study Group; CYTOL study group: Diagnostic value of biochemical markers (FibroTest-FibroSURE) for the prediction of liver fibrosis in patients with non-alcoholic fatty liver disease. BMC Gastroenterol 2006, 6:6.

10. Guha IN, Parkes J, Roderick P, Chattopadhyay D, Cross R, Harris S, Kaye P, Burt AD, Ryder SD, Aithal GP, Day CP, Rosenberg WM: Noninvasive markers of fibrosis in nonalcoholic fatty liver disease: validating the European Liver Fibrosis Panel and exploring simple markers. Hepatology 2008, 47:455-460.

11. Huwart L, Sempoux C, Salameh N, Jamart J, Annet L, Sinkus R, Peeters F, ter Beek $L C$, Horsmans $Y$, Van Beers BE: Liver fibrosis: noninvasive assessment with MR elastography versus aspartate aminotransferase- to-platelet ratio index. Radiology 2007, 245:458-466.

12. Yoneda M, Yoneda M, Mawatari H, Fuijta K, Endo H, lida H, Nozaki Y, Yonemitsu K, Higurashi T, Takahashi H, Kobayashi N, Kirikoshi H, Abe Y, Inamori M, Kubota K, Saito S, Tamano M, Hiraishi H, Maeyama S, Yamaguchi N, Togo S, Nakajima A: Noninvasive assessment of liver fibrosis by measurement of stiffness in patients with nonalcoholic fatty liver disease (NAFLD). Dig Liver Dis 2008, 40:371-378.

13. Angulo P, Hui JM, Marchesini G, Bugianesi E, George J, Farrell GC, Enders F, Saksena S, Burt AD, Bida JP, Lindor K, Sanderson SO, Lenzi M, Adams LA, Kench J, Therneau TM, Day CP: The NAFLD fibrosis score: a noninvasive system that identifies liver fibrosis in patients with NAFLD. Hepatology 2007, 45:846-854.

14. Ratziu V, Giral P, Charlotte F, Bruckert E, Thibault V, Theodorou I, Khalil L, Turpin G, Opolon P, Poynard T: Liver fibrosis in overweight patients. Gastroenterology 2000, 118:1117-1123.

15. Yoneda M, Suzuki K, Kato S, Fujita K, Nozaki Y, Hosono K, Saito S, Nakajima A: Nonalcoholic fatty liver disease: US-based acoustic radiation force impulse elastography. Radiology 2010, 256:640-647.

16. Suzuki A, Angulo P, Lymp J, Li D, Satomura S, Lindor K: Hyaluronic acid, an accurate serum marker for severe hepatic fibrosis in patients with nonalcoholic fatty liver disease. Liver Int 2005, 25:779-786.

17. Kaneda H, Hashimoto E, Yatsuji S, Tokushige K, Shiratori K: Hyaluronic acid levels can predict severe fibrosis and platelet counts can predict cirrhosis in patients with nonalcoholic fatty liver disease. $J$ Gastroenterol Hepatol 2006, 21:1459-1465.

18. Yoneda M, Mawatari H, Fujita K, Yonemitsu K, Kato S, Takahashi H, Kirikoshi H, Inamori M, Nozaki Y, Abe Y, Kubota K, Saito S, Iwasaki T, Terauchi Y, Togo S, Maeyama S, Nakajima A: Type IV collagen $7 \mathrm{~s}$ domain is an independent clinical marker of the severity of fibrosis in patients with nonalcoholic steatohepatitis before the cirrhotic stage. J Gastroenterol 2007, 42:375-381.

19. Harrison SA, Oliver D, Arnold HL, Gogia S, Neuschwander-Tetri BA: Development and validation of a simple NAFLD clinical scoring system for identifying patients without advanced disease. Gut 2008, 57:1441-1447.

20. Miyaaki H, Ichikawa T, Nakao K, Yatsuhashi H, Furukawa R, Ohba K, Omagari K, Kusumoto Y, Yanagi K, Inoue O, Kinoshita N, Ishibashi H, Yano M, Eguchi K: Clinicopathological study of nonalcoholic fatty liver disease in Japan: the risk factors for fibrosis. Liver Int 2008, 28:519-524.

21. Angulo P, Hui JM, Marchesini G, Bugianesi E, George J, Farrell GC, Enders F, Saksena S, Burt AD, Bida JP, Lindor K, Sanderson SO, Lenzi M, Adams LA, Kench J, Therneau TM, Day CP: The NAFLD fibrosis score: a noninvasive system that identifies liver fibrosis in patients with NAFLD. Hepatology 2007, 45:846-854.

22. Sterling RK, Lissen E, Clumeck N, Sola R, Correa MC, Montaner J, S Sulkowski M, Torriani FJ, Dieterich DT, Thomas DL, Messinger D, Nelson M, APRICOT Clinical Investigators: Development of a simple noninvasive index to predict significant fibrosis in patients with HIV/HCV coinfection. Hepatology 2006, 43:1317-1325.

23. Vallet-Pichard A, Mallet V, Verkarre V, Nalpas A, Dhalluin-Venier V, Fontaine $H$, Pol S: FIB-4: an inexpensive and accurate marker of fibrosis in HCV infection. Comparison with liver biopsy and fibrotest. Hepatology 2007, 46:32-36.

24. Shah A, Lydecker A, Murray K, Tetri BN, Contos MJ, Sanyal AJ, NASH Clinical Research Network: Comparison of noninvasive markers of fibrosis in patients with nonalcoholic fatty liver disease. Clin Gastroenterol Hepatol 2009, 7:1104-1112.

25. Sumida $Y$, Yoneda M, Hyogo H, Yamaguchi K, Ono M, Fujii H, Eguchi $Y$, Suzuki Y, Imai S, Kanemasa K, Fujita K, Chayama K, Yasui K, Saibara T, Kawada N, Fujimoto K, Kohgo Y, Okanoue T, Japan Study Group of Nonalcoholic Fatty Liver Disease (JSG-NAFLD): A simple clinical scoring system using ferritin, fasting insulin and type IV collagen $7 \mathrm{~S}$ for predicting steatohepatitis in nonalcoholic fatty liver disease. J Gastroenterol 2011, 46:257-268.

26. Kleiner DE, Brunt EM, Van Natta M, Behling C, Contos MJ, Cummings OW, Ferrell LD, Liu YC, Torbenson MS, Unalp-Arida A, Yeh M, McCullough AJ, Sanyal AJ, Nonalcoholic Steatohepatitis Clinical Research Network: Design and validation of a histological scoring system for nonalcoholic fatty liver disease. Hepatology 2005, 41:1313-1321.

27. Rickham PP: Human Experimentation. Code of ethics of the world medical association. Declaration of Helsinki. Br Med J 1964, 2:177.

28. Japanese Society for the Study of Obesity: New criteria of obesity (in Japanese). J Jpn Soc Study Obes 2000, 6:18-28.

29. American Diabetes Association: Report of the expert committee on the diagnosis and classification of diabetes mellitus. Diabetes Care 1997, 20:1183-1197.

30. Expert Panel on Detection, Treatment of High Blood Cholesterol in Adults: Executive Summary of The Third Report of The National Cholesterol Education Program (NCEP) Expert Panel on Detection, Evaluation, And Treatment of High Blood Cholesterol In Adults (Adult Treatment Panel III). JAMA 2001, 285:2486-2497.

31. Wai CT, Greenson JK, Fontana RJ, Kalbfleisch JD, Marrero JA, Conjeevaram HS, Lok AS: A simple noninvasive index can predict both significant fibrosis and cirrhosis in patients with chronic hepatitis $C$. Hepatology 2003, 38:518-526.

32. Poynard T, Bedossa P: Age and platelet count: a simple index for predicting the presence of histological lesions in patients with antibodies to hepatitis C virus. METAVIR and CLINIVIR Cooperative Study Groups. J Viral Hepat 1997, 4:199-208.

33. Fujii H, Enomoto M, Fukushima W, Tamori A, Sakaguchi H, Kawada N: Applicability of BARD score to Japanese patients with NAFLD. Gut 2009, 58:1566-1567.

34. Wong WW, Wong GL, Chim AM, Tse AM, Tsang SW, Hui AY, Choi PC, Chan AW, So WY, Chan FK, Sung JJ, Chan HL: Validation of the NAFLD fibrosis score in a Chinese population with low prevalence of advanced fibrosis. Am J Gastroenterol 2008, 103:1682-1688.

35. Fracanzani AL, Valenti L, Bugianesi E, Andreoletti M, Colli A, Vanni E, Bertelli C, Fatta E, Bignamini D, Marchesini G, Fargion S: Risk of Severe Liver Disease in Nonalcoholic Fatty Liver Disease with Normal Aminotransferase Levels: A Role for Insulin Resistance and Diabetes. Hepatology 2008, 48:792-798.

36. Mofrad P, Contos MJ, Haque M, Sargeant C, Fisher RA, Luketic VA, Sterling RK, Shiffman ML, Stravitz RT, Sanyal AJ: Clinical and Histologic Spectrum of Nonalcoholic Fatty Liver Disease Associated With Normal ALT Values. Hepatology 2003, 37:1286-1292.

37. Uslusoy HS, Nak SG, Gülten M, Biyikli Z: Non-alcoholic steatohepatitis with normal aminotransferase values. World J Gastroenterol 2009, 21:1863-1868.

\section{Pre-publication history}

The pre-publication history for this paper can be accessed here: http://www.biomedcentral.com/1471-230X/12/2/prepub

doi:10.1186/1471-230X-12-2

Cite this article as: Sumida et al: Validation of the FIB4 index in a Japanese nonalcoholic fatty liver disease population. BMC Gastroenterology 2012 12:2. 